\title{
Loss of HIPK2 Protects Neurons from Mitochondrial Toxins by Regulating Parkin Protein Turnover
}

\author{
(-) Jiasheng Zhang, ${ }^{1,2}$ Yulei Shang, ${ }^{1}$ Sherry Kamiya, ${ }^{1}$ Sarah J. Kotowski, ${ }^{3,4}$-Ken Nakamura, ${ }^{3,4}$ and $\odot$ Eric J. Huang ${ }^{1,2}$ \\ ${ }^{1}$ Department of Pathology, University of California, San Francisco, San Francisco, California 94143, ${ }^{2}$ Pathology Service 113B, VA Medical Center, San \\ Francisco, California 94121, ${ }^{3}$ Department of Neurology, University of California, San Francisco, San Francisco, California 94122, and ${ }^{4}$ Gladstone Institute of \\ Neurological Disease, San Francisco, California 94158
}

Mitochondria are important sources of energy, but they are also the target of cellular stress, toxin exposure, and aging-related injury. Persistent accumulation of damaged mitochondria has been implicated in many neurodegenerative diseases. One highly conserved mechanism to clear damaged mitochondria involves the E3 ubiquitin ligase Parkin and PTEN-induced kinase 1 (PINK1), which cooperatively initiate the process called mitophagy that identifies and eliminates damaged mitochondria through the autophagosome and lysosome pathways. Parkin is a mostly cytosolic protein, but is rapidly recruited to damaged mitochondria and target them for mitophagy. Moreover, Parkin interactomes also involve signaling pathways and transcriptional machinery critical for survival and cell death. However, the mechanism that regulates Parkin protein level remains poorly understood. Here, we show that the loss of homeodomain interacting protein kinase 2 (HIPK2) in neurons and mouse embryonic fibroblasts (MEFs) has a broad protective effect from cell death induced by mitochondrial toxins. The mechanism by which Hipk $2^{-1}$ neurons and MEFs are more resistant to mitochondrial toxins is in part due to the role of HIPK2 and its kinase activity in promoting Parkin degradation via the proteasome-mediated mechanism. The loss of HIPK2 leads to higher cytosolic Parkin protein levels at basal conditions and upon exposure to mitochondrial toxins, which protects mitochondria from toxin-induced damage. In addition, Hipk $2^{-1-}$ neurons and MEFs show increased expression of PGC- $1 \alpha$ (peroxisome proliferator-activated receptor- $\gamma$ coactivator 1 ), a Parkin downstream target that can provide additional benefits via transcriptional activation of mitochondrial genes. Together, these results reveal a previously unrecognized avenue to target HIPK2 in neuroprotection via the Parkin-mediated pathway.

Key words: HIPK2; mitochondria; mitophagy; Parkin; PGC-1; proteasome

\section{Significance Statement}

In this study, we provide evidence that homeodomain interacting protein kinase 2 (HIPK2) and its kinase activity promote Parkin degradation via the proteasome-mediated pathway. The loss of HIPK2 increases cytosolic and mitochondrial Parkin protein levels under basal conditions and upon exposure to mitochondrial toxins, which protect mitochondria from toxin-induced damage. In addition, Hipk $2^{-1-}$ neurons and mouse embryonic fibroblasts also show increased expression of PGC-1 $\alpha$ (peroxisome proliferator-activated receptor- $\gamma$ coactivator 1), a Parkin downstream target that can provide additional benefits via transcriptional activation of mitochondrial genes. These results indicate that targeting HIPK2 and its kinase activity can have neuroprotective effects by elevating Parkin protein levels.

\section{Introduction}

Mitochondria provide important sources of cellular energy via the execution of a wide range of functions, including respiratory complex assembly, ATP production, $\mathrm{Ca}^{2+}$ homeostasis and radical oxygen species (ROS) production. In addition, mitochondria interact with many intracellular organelles, such as endoplasmic reticulum (ER), autophagosomes, lysosomes, and microtubules,
Affairs or the United States Government. We thank Ivy Hsieh for assistance with electron microscopy, Viktorila Kolotovska for Seahorse XF Cell Mito Stress tests, and members of the Huang and Nakamura laboratories for feedback during the course of this study.

The authors declare no competing financial interests.

Correspondence should be addressed to Eric J. Huang at eric.huang2@ucsf.edu.

https://doi.org/10.1523/JNEUROSCI.2017-19.2019

Copyright $\odot 2020$ the authors 
to maintain cellular homeostasis (Sheng and Cai, 2012; Youle and van der Bliek, 2012). Dysfunctions in mitochondria have been implicated in neurodevelopmental disorders and neurodegenerative diseases, including Parkinson's disease, Alzheimer's disease, and amyotrophic lateral sclerosis. In addition to genetic mutations that directly disrupt mitochondrial quality control, there is compelling evidence that misfolded proteins associated with neurodegenerative diseases, including $\alpha$-synuclein, amyloid- $\beta$, tau, TDP-43 (TAR DNA-binding protein 43), and mutant SOD1 (superoxide dismutase 1) proteins, can also disrupt normal mitochondrial functions (Eckert et al., 2010; Israelson et al., 2010; Nakamura et al., 2011; Wang et al., 2016).

First identified in patients with familial Parkinson's disease, mutations in Parkin (PARK2) and PINK1 (PARK6) interfere with the normal functions of Parkin and PTEN-induced kinase 1 (PINK1) in the ubiquitination of the outer membrane of mitochondria to promote mitophagy, an important and evolutionarily conserved mitochondrial quality control process that identifies impaired mitochondria and removes them from the mitochondrial network (Narendra and Youle, 2011; Klein and Westenberger, 2012). Parkin is an E3 ubiquitin ligase that is predominantly cytosolic under basal conditions. However, when cells are treated with carbonyl cyanide m-chlorophenylhydrazone (CCCP), which uncouples mitochondrial membrane potentials by increasing the inner membrane permeability to protons, Parkin is rapidly recruited to the outer membrane of mitochondria (Narendra et al., 2008). Several lines of evidence indicate that PINK1 promotes Parkin recruitment to the mitochondria, and that both Parkin and PINK1 are required to promote the degradation of mitochondria via the autophagosome pathway (Matsuda et al., 2010; Vives-Bauza et al., 2010). In addition to chemical uncouplers, chronic oxidative stress and mitochondrial dysfunction can also promote Parkin recruitment to mitochondria.

In addition to its role in mitophagy, Parkin can affect mitochondrial function via a transcriptional mechanism. For instance, yeast two-hybrid screens identified human PARIS (ZNF746) as a Parkininteracting substrate that contains a Kruppel-associated box in its $\mathrm{N}$ terminus and a $\mathrm{C} 2 \mathrm{HC} / \mathrm{C} 2 \mathrm{H} 2$ zinc finger domain at its $\mathrm{C}$ terminus (Shin et al., 2011). Downregulation or inactivation of Parkin leads to increased PARIS levels, which suppress the transcription of peroxisome proliferator-activated receptor- $\gamma$ coactivator 1 (PGC- $1 \alpha$ ) and PGC- $1 \alpha$ downstream target genes that are critical for mitochondrial functions. Consistent with these results, the coexpression of Parkin and PGC- $1 \alpha$ increases the number of mitochondria, enhances mitochondrial respiration, and promotes the functional recovery of mitochondria after membrane uncoupling (Zheng et al., 2017). Despite the robust evidence supporting the role of Parkin in mitophagy, the exact mechanism that regulates the distribution of Parkin in the cytosol and its recruitment to mitochondria upon membrane uncoupling remains poorly understood. It is also unclear what controls Parkin protein levels and whether increasing the cytosolic Parkin protein level has any beneficial effect in protecting neurons from mitochondrial toxicity.

Homeodomain interacting protein kinase 2 (HIPK2) is a serine/threonine kinase that regulates tumorigenesis, neural development, and tissue fibrosis (Hofmann et al., 2013; Fan et al., 2014; Blaquiere and Verheyen, 2017). In our recent studies, we have shown that the loss of HIPK2 promotes neuronal survival when exposed to ER stress induced by misfolded SOD1 $193 \mathrm{~A}$ proteins or by mitochondrial toxin 1-methyl-4-phenyl-1,2,3,6tetrahydropyridine (MPTP; Lee et al., 2016; Shang et al., 2018). Here, we show that $H_{i p k 2^{-1-}}$ neurons have broader resistance to mitochondrial toxins and that such a neuroprotective effect is due in part to a persistent increase of Parkin protein levels in the cytosol and mitochondria under normal growth conditions and upon mitochondrial membrane uncoupling. We further show that HIPK2 promotes Parkin degradation via the proteasomemediated mechanism and that this process requires HIPK2 kinase activity. Consistent with the elevated Parkin protein level, Hipk $2^{-1-}$ neurons show increased expression of PGC- $1 \alpha$, which can activate the transcription of target genes that promote mitochondrial functions. Together, these results support the idea that targeting HIPK2 and its kinase activity may promote neuronal survival under stress conditions by regulating Parkin protein level.

\section{Materials and Methods}

Animals. Hipk2 $2^{-1-}$ mice (Hipk2 $2^{\text {tm1Ejh }} /$ Hipk2 $2^{\text {tm1Ejh }}$; RRID:MGI:5008273/ RRID:MGI:3510466) have been described previously (Wiggins et al., 2004; Zhang et al., 2007). Hipk2 $2^{+/+}$and Hipk2 ${ }^{-1-}$ mice in the mixed C67BL/6 and 129 background were used at 2 months old. TH-IRES-Cre mice $\left(T h^{t m 1(\text { cre }) T e}\right.$; catalog \#3056580, MGI) were described previously (Lindeberg et al., 2004; Tang et al., 2009). $R 26 R^{H I P K 2}$ mice were described previously (Shang et al., 2018) and maintained in a mixed C57BL/6 and 129 background. Mice of both sexes were selected and assigned to each age or treatment group randomly. Animal care was approved by the Institutional of Animal Care and Use Committee (IACUC) at the University of California, San Francisco (UCSF) and followed the National Institutes of Health (NIH) guidelines.

MPTP treatment in Hipk2 ${ }^{+/+}$, Hipk $2^{-/-}$, and TH-IRES-Cre; R26R ${ }^{\text {HIPK2/HIPK2 mice. Treatment of Hipk2 }}{ }^{+/+}, H_{i p k 2}{ }^{-1-}$, and $\mathrm{TH}_{-}$ IRES-Cre; $R 26 R^{H I P K 2 / H I P K 2}$ mice with MPTP was conducted according to previously published methods (Shang et al., 2018). Briefly, $4 \mathrm{mg} / \mathrm{kg}$ MPTP (catalog \#M103, Sigma-Aldrich) or an equivalent volume of PBS was injected intraperitoneally into 2-month-old male and female mice (littermates) by one injection per day for 10 consecutive days. The mice were monitored according to the approved IACUC protocol, and their health was scored before each injection. Seven days after the last MPTP treatment, mice were killed and perfused with $4 \%$ paraformaldehyde (PFA) before brain extraction. The brains were postfixed in $4 \%$ PFA overnight, followed by serial cryoprotection in 15 and $30 \%$ sucrose for $24 \mathrm{~h}$ each. The brains were embedded in O.C.T. (optimum cutting temperature) compound for cryosectioning and cut into $40 \mu \mathrm{m}$ coronal sections.

Electron microscopy and quantification of mitochondria morphology. Mice were deeply anesthetized with avertin $(150 \mathrm{mg} / \mathrm{kg})$ and were perfused transcardially with $2 \%$ PFA/ $0.2 \%$ glutaraldehyde in $0.1 \mathrm{M}$ phosphate buffer at $\mathrm{pH} 7.4$ with gravity. Brains were quickly removed, fixed overnight in $2 \%$ PFA at $4^{\circ} \mathrm{C}$, and cut into $60-\mu \mathrm{m}$-thick frontal sections with a vibratome. Finally, the sections were postfixed in $0.5 \%$ osmium tetroxide for $10 \mathrm{~min}$, dehydrated, and embedded in resin (Durcupan ACM). To identify dopaminergic (DA) neurons to electron microscopic analysis, we prepared $100 \mu \mathrm{m}$ brain sections at the level of $-2.75 \mathrm{~mm}$ from bregma from Hipk2 $2^{+/+}$and Hipk2 $2^{-1-}$ mice. The substantia nigra was then dissected using a Nikon stereomicroscope. Serial ultrathin sections of Hipk2 $2^{+/+}$and Hipk2 $2^{-1-}$ substantia nigra were cut with a Reichert Ultracut $S$ microscope, contrasted with lead citrate. Ultrathin sections were cut at a thickness of $1 \mu \mathrm{m}$ with a Reichert Ultracut $S$ microscope, contrasted with lead citrate, and imaged in a Philips Tecnai10 transmission electron microscope using FEI software. Mitochondrial morphology was characterized by measuring the ferret diameter and surface area of mitochondria in DA neurons in the substantia nigra of control and MPTP-treated Hipk2 ${ }^{+/+}, H_{i p k}{ }^{-1-}$, and TH-IRES-Cre;R26R ${ }^{H I P K 2 / H I P K 2}$ mice. The measurements were performed in three mice per genotype and mitochondria in $10 \mathrm{DA}$ neurons were selected for quantification.

Primary dopaminergic neuron cultures and mouse embryonic fibroblasts. Primary DA neurons were prepared according to published procedures (Zhang et al., 2007; Tang et al., 2010; Luo et al., 2016). Briefly, embryonic 
day 13.5 (E13.5) Hipk2 ${ }^{+/+}$and Hipk2 ${ }^{-1-}$ mouse embryos were collected from time-pregnant $H i p k 2^{+l-}$ females. The ventral mesencephalon was dissected, dissociated after treatment with trypsin, and cultured in Invitrogen DMEM-F12 medium (catalog \#11765-054, Thermo Fisher Scientific) supplemented with Gibco $10 \%$ FBS, $1 \times$ penicillin/streptozhymycin, and 2 mm glutamine (Thermo Fisher Scientific) on cover slides coated with poly-DL-ornithine hydrobromide (catalog \#P8638, SigmaAldrich) overnight and laminin (catalog \#L2020, Sigma-Aldrich) for $2 \mathrm{~h}$. On DIV1, the medium was replaced with Gibco DMEM-F12 medium with $1 \times$ N2 supplement (catalog \#17-502-048, Thermo Fisher Scientific), $20 \mathrm{ng} / \mathrm{ml} \mathrm{FGF2,} 100 \mathrm{ng} / \mathrm{ml} \mathrm{FGF8}$, and $1 \times$ penicillin/streptomycin for $2 \mathrm{~d}$. To characterize neurotoxic responses, Hipk2 ${ }^{+/+}$and Hipk2 ${ }^{-1-}$ DA neurons were treated with 1-methyl-4-phenylpyridinium $\left(\mathrm{MPP}^{+} ; 5\right.$ $\mu \mathrm{M}$; catalog \#D048, Sigma-Aldrich,), rotenone (0.25, 0.5, 1, 2.5, or 5 nм; catalog \#R8875, Sigma-Aldrich), paraquat dichloride (1,1'-dimethyl4,4'-bipyridinium dichloride; $5,10,25$, or $50 \mu \mathrm{M}$; catalog \#856177, Sigma-Aldrich), or CCCP $(0.25,0.5,1,2.5$, or 5 nM; catalog \#C2759, Sigma-Aldrich) on DIV3 for $24 \mathrm{~h}$. As a control, each treatment regimen included identical primary neuron cultures where the neurons were incubated with control media without any neurotoxin. After $24 \mathrm{~h}$ of incubation, cultured neurons were fixed with $4 \%$ PFA for $10 \mathrm{~min}$, and stained with anti-TH antibody (catalog \#AB152, Millipore Bioscience Research Reagents) and anti-Tuj1 antibody (catalog \#PRB-435P, Covance) following the standard straining procedures. Confocal images of the cultured neurons were captured using a Leica confocal microscope (TCS $\mathrm{SP}$ ). Laser intensity (measured as the PMT levels) for each fluorophore was kept within the linear range. $\mathrm{TH}^{+}$or $\mathrm{TuJ} 1^{+}$Cell number was determined by NIH ImageJ online software. The percentage of surviving neurons was calculated using the total number of $\mathrm{TH}^{+}$or $\mathrm{TuJ} 1^{+}$neurons in neurotoxin-treated coverslips divided by the total number of $\mathrm{TH}^{+}$or $\mathrm{TuJ} 1^{+}$neurons in control DMSO-treated coverslips. Data represented results from four independent biological replicates.

Measurements for Parkin recruitment to mitochondria, mitochondrial mass, membrane potentials, and energetics using Seahorse Extracellular Flux Cell Mito Stress Test. Hipk2 ${ }^{+/+}$and Hipk2 $2^{-1-}$ mouse embryonic fibroblasts (MEFs) and HEK293 cells have been reported in our previous studies (Wei et al., 2007; Lee et al., 2016; Shang et al., 2018). To characterize Parkin recruitment to mitochondria, Hipk2 $2^{+/+}$and Hipk2 $2^{-1-}$ MEFs were transfected with FLAG-Parkin cDNA using Lipofectamine (catalog \#L3000-015, Thermo Fisher Scientific). Twenty-four hours after transfection, cells were treated with DMSO or $5 \mu \mathrm{M}$ CCCP for $2 \mathrm{~h}$, fixed in $4 \%$ PFA for $30 \mathrm{~min}$, and processed for immunofluorescent staining with anti-FLAG M2-monoclonal antibody (1:1000 dilution; catalog \#F3165, Sigma-Aldrich) and anti-Tom20 antibody (1:1000 dilution; catalog \#sc-11415, Santa Cruz Biotechnology). Images of these cells were captured using a Nikon C2 Confocal Microscope. Parkin and Tom20 fluorescent signal intensity was measured using NIS-Elements software.

To measure mitochondrial mass, MitoTracker Green FM Dye (catalog \#M7514, Thermo Fisher Scientific) and MitoTracker Red FM Dye (catalog \#M22425, Thermo Fisher Scientific) were added to Hipk $2^{+/+}$and Hipk $2^{-1-}$ MEFs according to manufacturer instructions. Afterward, Hipk $2^{+/+}$and Hipk2 ${ }^{-1-}$ MEFs were treated with DMSO or $5 \mu \mathrm{M}$ CCCP for 6 or $24 \mathrm{~h}$, and collected for fluorescence-activated cell sorting (FACS) analyses using FACSAria III (BD Biosciences) at UCSF Parnassus Flow Cytometry Core. Respiratory and glycolytic rates in $\mathrm{Hipk}^{+/+}$and Hipk2 ${ }^{-1-}$ MEFs were measured using the Seahorse Extracellular Flux (XF) Analyzer 96-well plate reader (Agilent Technologies) as previously described (Mendelsohn et al., 2018). Briefly, Hipk2 $2^{+1+}$ and Hipk2 ${ }^{-1-}$ MEFs were seeded at 150,000 cells/well in Seahorse assay medium, supplemented with $10 \mathrm{~mm}$ pyruvate in 96-well plates precoated with 22.4 $\mu \mathrm{g} / \mathrm{ml}$ Cell-Tak and Tissue Adhesive (catalog \#CB40240, Corning). Respiration and glycolysis were simultaneously measured based on oxygen consumption rates (OCRs) and extracellular acidification consumption rates (ECARs), respectively. OCR and ECAR were measured three times before injection and three times after sequential injection of $2 \mu \mathrm{g} / \mathrm{ml}$ oligomycin or $2 \mu \mathrm{M}$ carbonyl cyanide-4-(trifluoromethoxy) phenylhydrazone (FCCP; catalog \#C2920, Sigma-Aldrich). The measurements at each time point were normalized to the value of the first time point on a well-by-well basis.
Protein lysate preparation, mitochondria isolation and Western blot analysis. Mitochondria were isolated from Hipk2 $2^{+/+}$and Hipk2 $2^{-1-}$ brains and MEFs using Mitochondria Isolation Kits for tissues and cells (catalog \#89801 and \#89874, respectively, Thermo Fisher Scientific). Protein lysates were prepared from Hipk2 $2^{+/+}$and Hipk2 ${ }^{-1-}$ MEFs or mitochondria isolated from these cells using NP-40 lysis buffer (1\% NP40, 20 mм Tris, pH 7.6, $150 \mathrm{~mm} \mathrm{NaCl,} 10 \mathrm{~mm} \mathrm{NaF,} 1$ mм $\mathrm{Na}_{3} \mathrm{VO}_{4}$ ) supplemented with protease inhibitor cocktail. To prepare protein lysates from brain tissues, we first microdissected substantia nigra or cerebral cortex from 2-month-old Hipk2 $2^{+/+}$and Hipk2 $2^{-1-}$ mice and lysed the tissues using RIPA buffer $(0.1 \%$ SDS, $1 \%$ sodium deoxycholate, $1 \%$ NP-40, 20 mm Tris, pH 7.6, $150 \mathrm{~mm} \mathrm{NaCl}, 10 \mathrm{~mm} \mathrm{NaF}, 1$ mм $\mathrm{Na}_{3} \mathrm{VO}_{4}$ ) supplemented with protease inhibitor cocktail. Protein lysates were separated by SDS-PAGE and transferred to the PVDF membrane (Millipore). The membrane was blocked in 4\% BSA (for phosphor antibodies) or $5 \%$ nonfat milk for nonphosphorylated antibodies before being incubated with primary antibodies overnight at $4^{\circ} \mathrm{C}$. The sources and conditions for the primary antibodies were as follows: anti-Parkin antibody (1:2000 dilution for Western blot; catalog \#ab15954, Abcam), anti-FLAG M2 antibody (1:1000 for Western blot; catalog \#F3165, Sigma-Aldrich), anti-NeuN antibody [1:500 dilution for immunofluorescence (IF) microscopy; catalog \#ABN78, Millipore], anti-PGC-1 $\alpha$ antibody (1:1000 dilution for Western blot and IF microscopy; catalog \#KP9803, Calbiochem), anti-Tom20 antibody (1:5000 for Western blot; catalog \#sc11415, Santa Cruz Biotechnology), anti-VDAC antibody (1:1000 dilution for Western blot; catalog \#4661s, Cell Signaling Technology), anti-cytochrome $c$ antibody (1:500 for IF microscopy; catalog \#556432, sBD Biosciences), and anti-actin antibody (1:5000 dilution for Western blot; catalog \#CP01, Calbiochem). The membranes were washed with $0.1 \%$ TBST washing buffer followed by incubation with secondary antibodies conjugated with horseradish peroxidase. Western blots were developed by ECL Chemiluminescence (catalog \#32132, Thermo Fisher Scientific).

Quantification of Western blot results was performed by normalizing the protein of interest to the loading controls, which included actin for cytosolic proteins and Tom20. Or VDAC1 for mitochondrial proteins. The normalized protein levels were then compared with those in Hipk2 ${ }^{+/+}$ or basal conditions to obtain the fold change between Hipk $2^{+/+}$and Hipk $2^{-1-}$ (see Figs. 3B, 6C) or different experimental conditions (see Fig. $3 D$ ). The quantitative analysis seen in Figure $4 J$ was performed by using a normalized Parkin level in the cytosol of Hipk2 ${ }^{+/+}$MEFs treated with DMSO.

Experimental design and statistical analyses. For both in vivo and in vitro studies, at least three biological replicates were used in each study. Experiments in which $N$ was $>3$, the exact number of replicates will be indicated. All data were expressed as the mean \pm SEM. Data were analyzed using the following statistical analyses with Prism (GraphPad Software): two-tailed Student's $t$ test (Figs. $1 B ; 2 B, C ; 3 B, D ; 4 I, K ; 5 C, E$; and $6 B, D$ ); and two-way ANOVA (Fig. $1 C-H$ ).

Data were used to compare the differences between the mean values $\left.{ }^{*} p<0.05,{ }^{* *} p<0.01,{ }^{* *} p<0.001\right) ; p>0.05$ is considered nonsignificant (n.s.).

\section{Results}

\section{Loss of HIPK2 protects neurons from mitochondrial toxins}

Our previous results showed that Hipk2 ${ }^{-1-}$ DA neurons are more resistant to mitochondrial toxin MPTP, whereas overexpressing HIPK2 in DA neurons using TH-IRES-Cre;R26R ${ }^{H I P K 2 / H I P K 2}$ mice enhances MPTP-induced cell death (Shang et al., 2018). Given the effects of MPTP as a mitochondrial complex I inhibitor, we asked whether the loss of HIPK2 may have broader protective effects on mitochondrial toxins. To test this, we established primary DA neuron cultures from the ventral mesencephalon of Hipk $2^{+/+}$and Hipk2 $2^{-1-}$ mice at E13.5. Consistent with the in vivo data, $\mathrm{TH}^{+} ; \mathrm{TuJ1}{ }^{+} \mathrm{DA}$ neurons from Hipk2 ${ }^{-1-}$ mice were more resistant to $\mathrm{MPP}^{+}$-induced toxicity (65\% survival in Hipk $2^{-1-}$ DA neurons vs $12 \%$ in Hipk $2^{+/+}$DA neurons; Fig. $1 A, B)$. Interestingly, a direct comparison between $\mathrm{TH}^{-}$;Tuj1 ${ }^{+}$ 
A

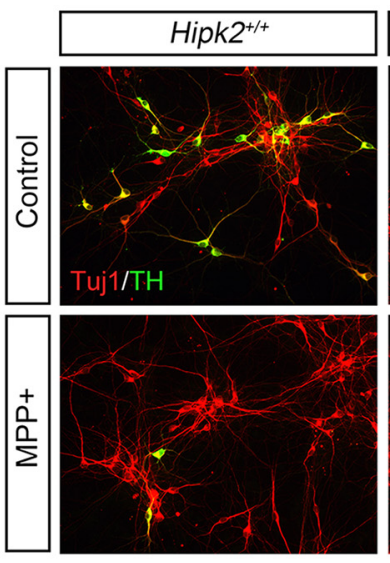

C

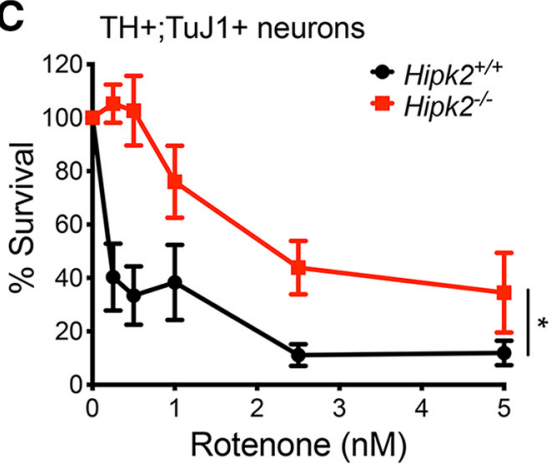

D

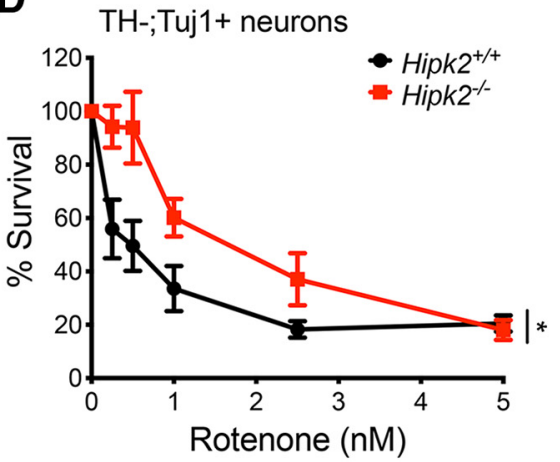

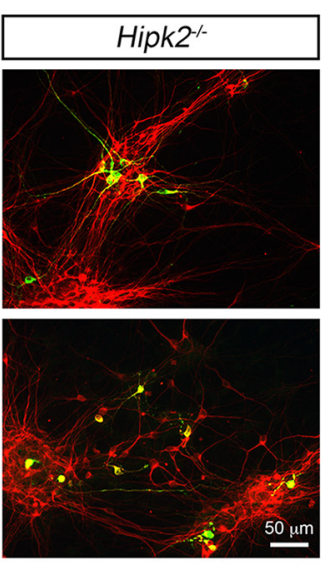

E

E TH+;TuJ1+ neurons

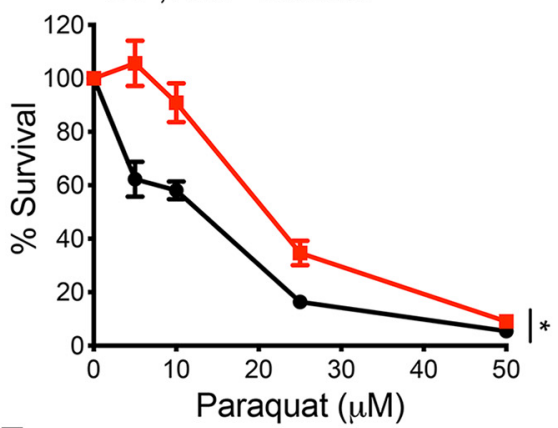

F

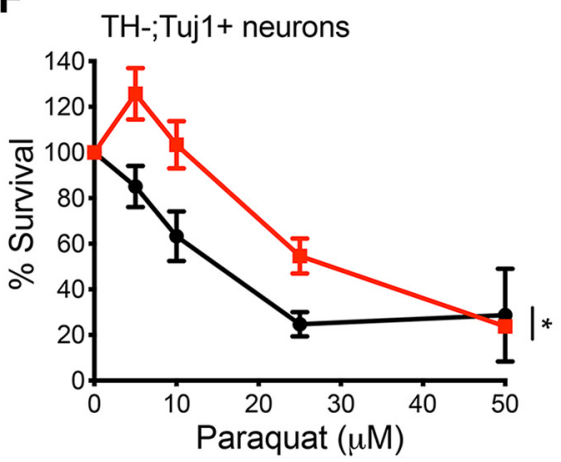

B $\mathrm{Hipk}^{+/+}$(control) $\mathrm{Hipk}^{-/-}$(control)

$H_{i p k 2^{+/+}+\mathrm{MPP}^{+} \quad \text { Hipk2 }}{ }^{-/-}+\mathrm{MPP}+$

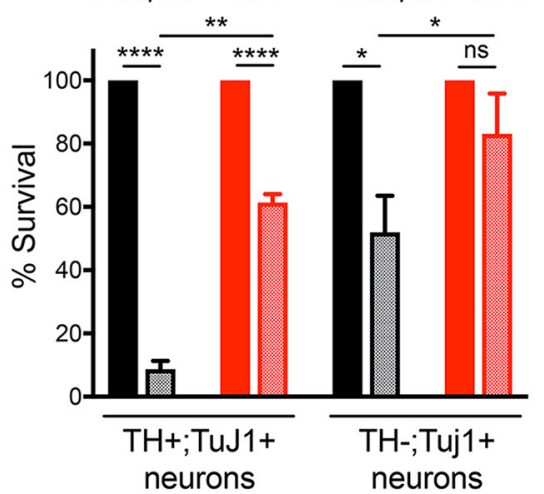

neurons

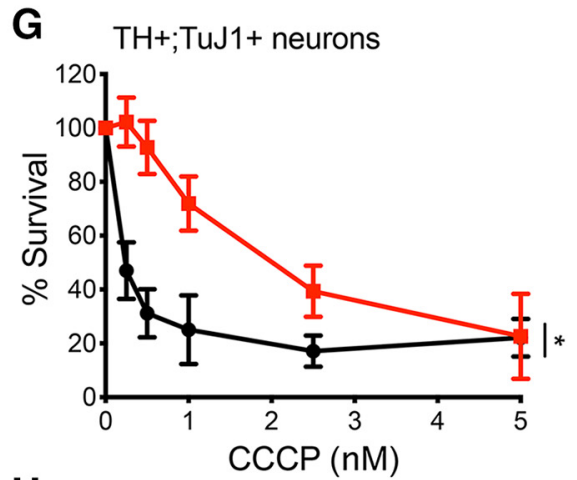

H

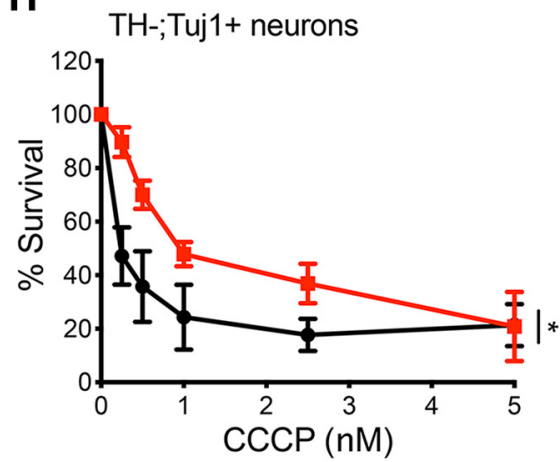

Figure 1. Hipk $2^{-I}$ neurons are more resistant to mitochondrial toxins. $A$, Immunofluorescent confocal microscopic images of primary neuron cultures using ventral mesencephalon of E13.5 Hipk2 ${ }^{+/+}$and Hipk2 $2^{-1-}$ embryos. The primary neurons, immunostained with anti-TH (green) and anti-TuJ1 (red) antibodies, were treated with DMSO (control) or MPP ${ }^{+}$(5 $\mu \mathrm{M})$ for $24 \mathrm{~h}$ before they were fixed and processed for image analyses. B, Quantification of $\mathrm{TH}^{+} ; \mathrm{TuJ}^{+}{ }^{+}$or $\mathrm{TH}^{-} ; \mathrm{TuJ}^{+}{ }^{+}$neurons from the ventral mesencephalon of E13.5 Hipk2 ${ }^{+/+}$and Hipk2 ${ }^{-1-}$ embryos showed that Hipk2 ${ }^{-1-} \mathrm{TH}^{+} ; \mathrm{TuJ}^{+}$and $\mathrm{TH}^{-} ; \mathrm{TuJ1}^{+}$neurons were more resistant to MPP ${ }^{+}$-induced toxicity. For each treatment paradigm, we set up one additional neuron culture without any neurotoxin treatment, which was considered as 0 concentration, and the percentage of survival in these cultures was counted as $100 \%$. Data represented the mean \pm SEM. Statistics used the Student's $t$ test: ns, not significant; ${ }^{*} p<0.05$; ${ }^{* *} p<0.01$; and ${ }^{* * * *} p<0.001$. C $-H$, Dose-response curves for Hipk2 ${ }^{+/+}$and Hipk2 ${ }^{-1-} \mathrm{TH}^{+} ; \mathrm{Tuj}^{+}{ }^{+}$and $\mathrm{TH}^{-} ; \mathrm{TuJ1}^{+}$neurons treated with rotenone $(0.25,0.5,1,2.5$, or $5 \mathrm{~nm})$, paraquat $(5,10,25$, or $50 \mu \mathrm{M})$, or $\mathrm{CCCP}(0.25,0.5,1,2.5$, or $5 \mathrm{~nm})$. Data represented the mean \pm SEM. Statistical analyses used a two-way ANOVA: ${ }^{*} p<0.05$.

Hipk $2^{+/+}$and Hipk2 $2^{-1-}$ neurons showed that Hipk2 $2^{-1-}$ non-DA neurons were also more resistant to $\mathrm{MPP}^{+}$(Fig. $1 B$ ), suggesting that the loss of HIPK2 might have more general neuroprotective effects toward mitochondrial toxicity. To test this, we treated Hipk $2^{+/+}$and Hipk2 $2^{-1-}$ primary neurons with additional mitochondrial toxins, rotenone, paraquat, and CCCP, which inhibits complex I, generates excess superoxide radicals, or uncouples oxidative phosphorylation, respectively (Li et al., 2003; Castello et al., 2007). Consistent with the results from $\mathrm{sMPP}^{+}$, both Hipk $2^{-1-}$ DA and non-DA neurons showed dose-dependent resistance to these mitochondrial toxins (Fig. $1 C-H$ ).

To further characterize the resistance of Hipk2 ${ }^{-1-}$ neurons to MPTP, we examined the mitochondrial morphology in the substantia nigra DA neurons of 2-month-old Hipk2 ${ }^{+/+}$, Hipk $2^{-1-}$, and TH-IRES-Cre;R26R ${ }^{H I P K 2 / H I P K 2}$ mice. In control PBS-injected mice, the mitochondrial morphology in the substantia nigra DA neurons of $\mathrm{Hipk}^{+/+}, \mathrm{Hipk}^{-{ }^{-}}$, and TH-IRES-Cre; R26R $R^{H I P K 2 / H I P K 2}$ mice showed no significant differences in the maximal diameter or surface area (Fig. 2A-C). However, following MPTP treatment, the mitochondrial diameter and surface area in Hipk2 ${ }^{+/+}$and TH-IRES-Cre; $R 26 R^{H I P K 2 / H I P K 2}$ neurons were significantly enlarged with the dissolution of cristae and the disruption of membrane integrity (Fig. $2 A$ ). In contrast, the mitochondria in the substantia nigra DA neurons in Hipk2 ${ }^{-1-}$ mice showed no significant enlargement or disruption of morphology. Together, these 
A
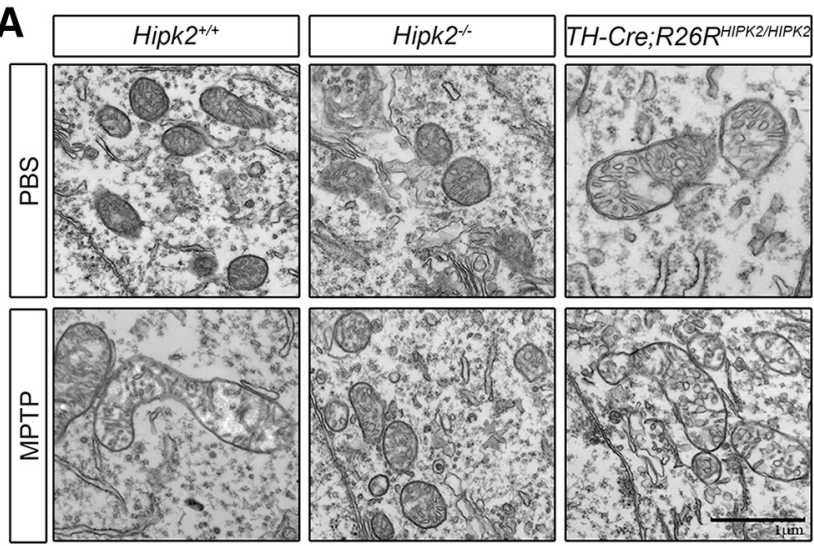

B
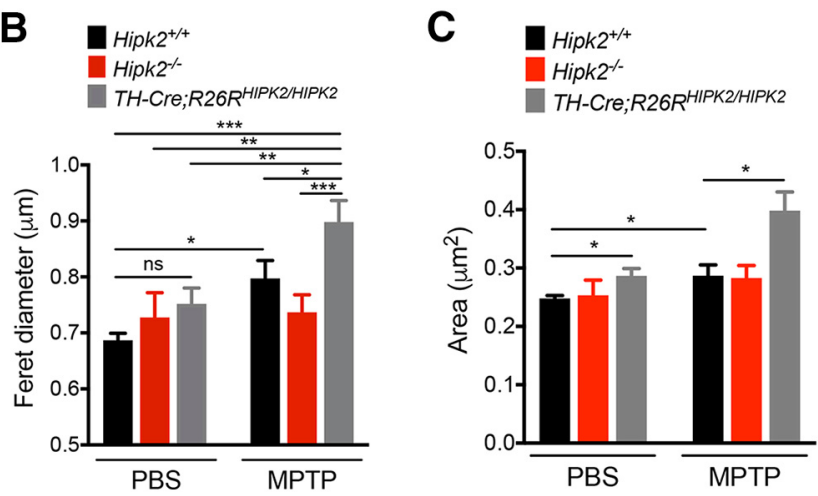

Figure 2. Effects of MPTP on the mitochondrial morphology of DA neurons in Hipk2 $^{+/+}$, Hipk2 ${ }^{-1-}$, and TH-IRES-Cre;R26R $R^{\text {HIPK2/HIPK2 }}$ mice. A, Transmission electron microscopic images of mitochondria in DA neurons in 2-month-old Hipk2 ${ }^{+/+}$, Hipk2 ${ }^{-1-}$, and TH-IRES-Cre;R26R $R^{\text {HIPK2/HIPK2 }}$ mice injected with PBS or MPTP (for detailed experimental procedures, see Materials and Methods). B, C, Quantification of mitochondrial morphology by measuring the diameter and area. Four mice per genotype and 10 neurons in the substantia nigra per mouse were used for this quantification. Data represented the mean \pm SEM. Statistical analysis used the Student's $t$ test: ns, not significant; ${ }^{*} p<0.05 ;{ }^{* *} p<0.01$; and ${ }^{* * *} p<0.005$.

results support the idea that loss of HIPK2 renders neurons more resistant to mitochondrial toxins.

\section{Loss of HIPK2 increases Parkin protein level via proteasome-mediated mechanism}

The resistance of Hipk2 ${ }^{-1-}$ neurons to mitochondrial toxins raises the possibility that the loss of HIPK2 may alter the protein constituents in the mitochondrial membranes to protect neurons from initiating the cell death process. To test this, we characterized a number of proteins that have been previously shown to regulate mitochondrial membrane potentials and responses to cell death signals, including Bcl-2, Bcl-xL, NRF1 (nuclear respiratory factor 1), Parkin, and PARIS, in Hipk2 ${ }^{+/+}$and Hipk2 $2^{-1-}$ mouse brains (Riedl and Salvesen, 2007; Chipuk and Green, 2008; Youle and Strasser, 2008). Among these candidates, a consistent increase in Parkin protein level in was detected in the lysates from the substantia nigra and cerebral cortex of Hipk2 $2^{-1-}$ mice (Fig. 3A). In support of these results, we isolated mitochondria from Hipk2 $2^{+/+}$and Hipk2 $2^{-1-}$ mouse brain and showed that the Parkin protein level was also significantly elevated in the mitochondria isolated from Hipk2 ${ }^{-1-}$ mouse brain (Fig. $3 A, B$ ). No difference was detected in the level of Bcl-xL, NRF1, or PARIS (Fig. 3C).

The elevated Parkin protein level in multiple regions of Hipk2 $2^{-1-}$ mouse brain raised the possibility that HIPK2 may regulate Parkin via transcription or post-translational mechanism. Since our previous transcriptomic analyses showed no difference in parkin mRNA level in Hipk2 ${ }^{-1-}$ mouse brain (Shang et al., 2018), we asked whether HIPK2 may regulate Parkin protein turnover via proteasome-mediated degradation. To this end, we expressed FLAG-tagged Parkin and HIPK2 in HEK293 cells and determined Parkin protein levels in these cells using Western blots. Our results showed that wild-type HIPK2 reduced Parkin protein level by $\sim 50 \%$, whereas kinase-inactive HIPK2 ${ }^{\mathrm{K} 221 \mathrm{~A}}$ did not consistently affect Parkin protein levels (Fig. $3 D, E$ ). Interestingly, the effects of wild-type HIPK2 to promote the reduction in Parkin was blocked by proteasome inhibitor MG132. Together, these results support the idea that HIPK2 regulates Parkin protein level via proteasome-mediated mechanisms and that this activity requires HIPK2 kinase activity.

\section{Elevated Parkin protein level in the mitochondria of Hipk2 ${ }^{-1-}$ MEF}

Given the elevated Parkin protein levels in Hipk2 $2^{-1-}$ mouse brain tissues, we asked how this might affect mitochondrial morphology during CCCP-induced membrane uncoupling. To test this, we expressed FLAG-Parkin in Hipk2 ${ }^{+/+}$and Hipk2 $2^{-1-}$ MEFs and treated these cells with DMSO or $5 \mu \mathrm{M}$ CCCP. In control DMSO-treated conditions, Parkin fluorescent intensity in the cytoplasm and mitochondria was consistently higher in $H i p k 2^{-1-}$ MEF than that in Hipk2 $2^{+/+}$MEF (Fig. 4A, B, E, F,I). Upon CCCP treatment, Hipk2 $2^{+/+}$MEF showed rapid recruitment of Parkin to Tom $20^{+}$mitochondria and a marked reduction in overall cytoplasmic Parkin protein level (Fig. 4C,D). However, unlike Hipk2 ${ }^{+/+}$MEF, Hipk2 ${ }^{-/-}$MEF continued to have a high abundance of Parkin in the cytoplasm, similar to the level at basal conditions (Fig. 4G,H). Consistent with these results, quantification of Parkin fluorescent intensity indeed confirmed the higher Parkin protein levels in Hipk2 ${ }^{-1-}$ MEF compared with Hipk2 ${ }^{+/+}$MEF (Fig. 4I).

To further characterize the subcellular distribution of Parkin, we used Western blots to characterize Parkin protein levels in the cytosol and mitochondria from Hipk2 ${ }^{+/+}$and Hipk2 ${ }^{-1-}$ MEFs that had been treated with DMSO or CCCP. Consistent with the results from immunofluorescent microscopy, Western blots using protein lysates from the cytosol showed that the basal Parkin protein level in the cytosol of Hipk2 $2^{-1-}$ MEF was higher than that in $\mathrm{Hipk}^{+/+}$MEF (Fig. 4J,K). Upon CCCP treatment, Hipk2 $^{+/+}$MEF showed a modest reduction in Parkin protein level in the cytosol, whereas Hipk2 ${ }^{-1-}$ MEF showed a significant increase of Parkin in the cytosol. Western blot analyses using mitochondria from Hipk2 $2^{+/+}$and Hipk2 $2^{-1-}$ MEF showed that under control DMSO treatment condition Parkin protein level was low in $\mathrm{Hipk}^{+/+}$MEF, but CCCP treatment induced Parkin recruitment to the mitochondria in Hipk2 ${ }^{+/+}$MEF (Fig. 4J,K). In contrast to Hipk2 ${ }^{+/+} \mathrm{MEF}$, mitochondria in Hipk2 ${ }^{-l_{-}^{-}}$MEF showed significantly higher basal Parkin protein level in the mitochondria before and after CCCP treatment (Fig. $4 \mathrm{~J}, \mathrm{~K}$ ). Together, these results support the idea that the loss of HIPK2 results in consistently high Parkin protein levels in the cytosol and mitochondria at basal level and after CCCP-induced mitochondrial membrane uncoupling.

\section{Mitochondria in Hipk2 $2^{-/-}$MEFs are resistant to chemical-induced membrane uncoupling}

To characterize how the loss of HIPK2 affects the mitochondrial membrane potentials, we incubated Hipk2 $2^{+/+}$and Hipk2 $2^{-1-}$ 
A

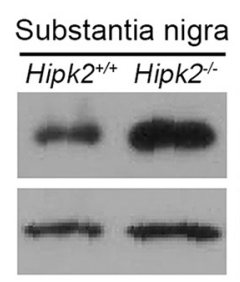

Brain mitochondria

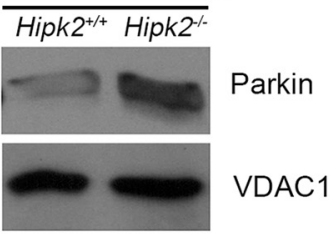

B

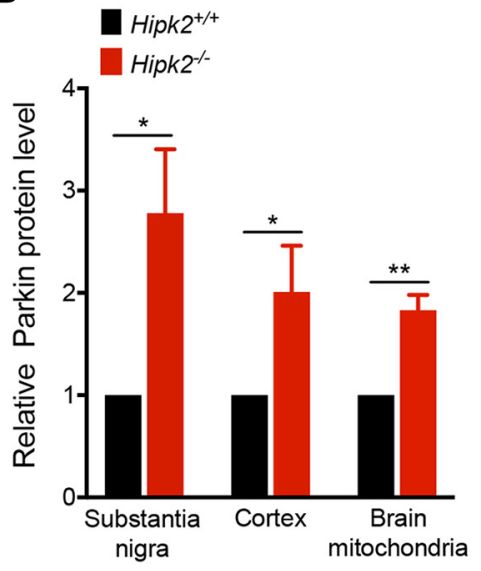

C

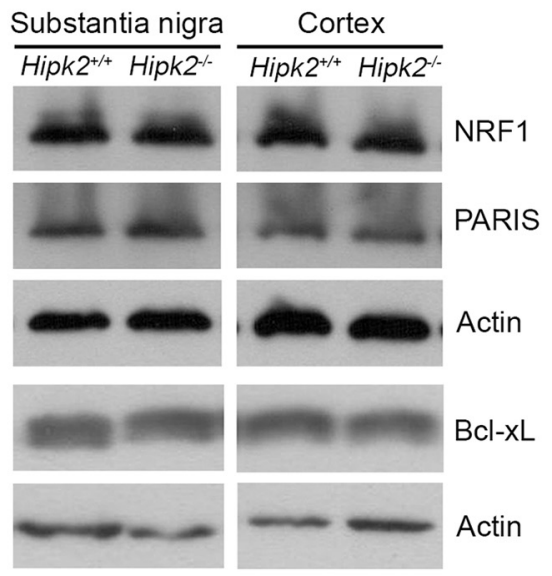

D

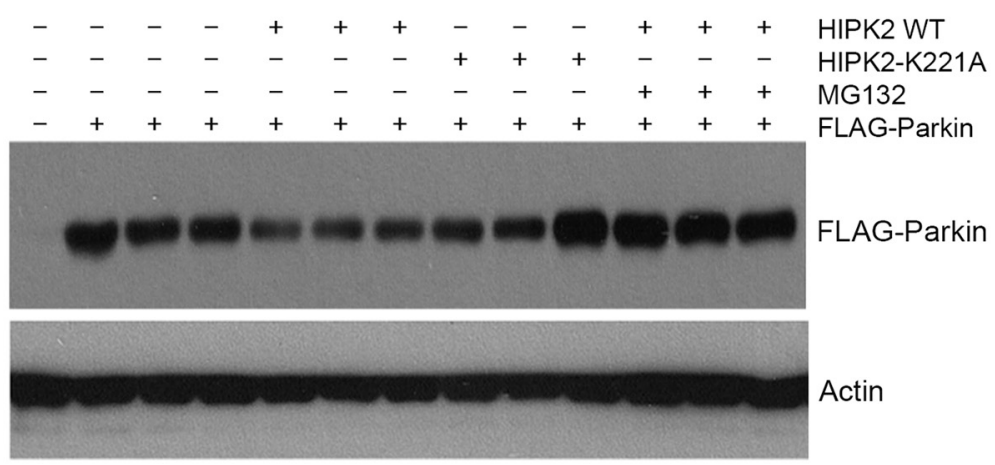

$\mathbf{E}$

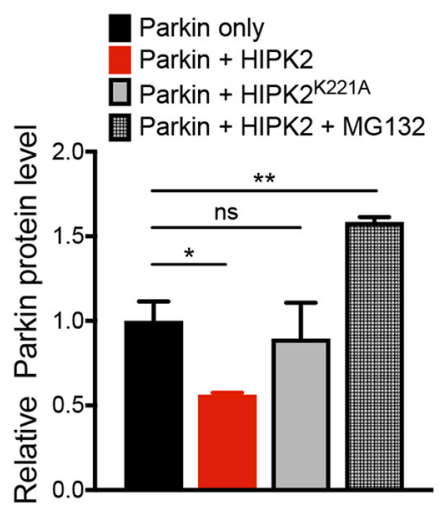

Figure 3. Loss of HIPK2 leads to elevated Parkin protein levels in Hipk2 $2^{-1-}$ brain tissues, whereas HIPK2 overexpression promotes Parkin degradation via the proteasome pathway. $A$, Western blot analyses detected Parkin protein levels in Hipk2 ${ }^{+/+}$and Hipk $2^{-1-}$ mouse substantia nigra and cerebral cortex. To characterize Parkin protein levels in mitochondria, fresh brain tissues from $H i p k 2^{+/+}$and Hipk2 ${ }^{-1-}$ mice were homogenized and mitochondria were isolated using the Mitochondria Isolation Kit (Thermo Fisher Scientific). B, Quantification of the relative abundance of Parkin protein levels in the substantia nigra, cerebral cortex, and brain mitochondria from Hipk2 $2^{+/+}$and Hipk2 $2^{-1-}$ mice. Data represented the mean \pm SEM. Statistical analyses used a Student's $t$ test: ${ }^{*} p<0.05$ and ${ }^{* *} p<0.01$. C, Western blot results showed no difference in the relative abundance of NRF1, PARIS, or BCl-xL in protein lysates in the substantia nigra or cortex of Hipk2 ${ }^{+/+}$ and Hipk2 $2^{-1-}$ mouse brain. D, Western blot analysis showed that HIPK2 overexpression reduced Parkin protein levels in HEK293 cells, and that this effect was not detected in kinase-inactive HIPK2, HIPK2 ${ }^{\text {K221A }}$. Treatment with proteasome inhibitor MG132 $(10 \mu \mathrm{M}, 4 \mathrm{~h})$ blocked the effect of HIPK2 to promote Parkin degradation. E, Quantification of relative Parkin protein levels in HEK293 cells transfected with FLAG-Parkin + wild-type HIPK2, FLAG-Parkin + HIPK2 ${ }^{\text {K221A }}$, or FLAG-Parkin + wild-type HIPK2 + MG132. Data represented the mean \pm SEM. Statistical analyses used the Student's t test: ns, not significant; ${ }^{*} p<0.05 ;{ }^{* *} p<0.01$.

MEFs with tetramethylrhodamine methyl ester (TMRM), a vital dye that detected mitochondrial membrane potentials, followed by sequential treatments with $2 \mu \mathrm{g} / \mathrm{ml}$ oligomycin to inhibit ATP synthase in complex V and $2 \mu \mathrm{M}$ FCCP to disrupt the proton gradient in mitochondrial membrane potential. This approach revealed no difference in TMRM signal intensity between Hipk $2^{+/+}$ and Hipk2 ${ }^{-1-}$ MEFs following oligomycin or FCCP treatment up to $10 \mathrm{~min}$ (Fig. 5A). Furthermore, Seahorse mitochondrial stress assays showed no difference in OCRs or ECARs between Hipk $2^{+/+}$and Hipk2 $2^{-1-}$ MEFs (data not shown).

To further determine how the loss of HIPK2 affects mitochondrial membrane potentials and integrity, we incubated Hipk $2^{+/+}$ and Hipk2 ${ }^{-1-}$ MEFs with MitoTracker Red and MitoTracker Green, which measured mitochondrial membrane potential and the mitochondrial mass by labeling the inner membrane, respectively. After incubation with these dyes, Hipk $2^{+/+}$and Hipk2 $2^{-1-}$ MEFs were treated with $5 \mu \mathrm{M}$ CCCP and collected at 2, 6, and $24 \mathrm{~h}$ after treatment for FACS analyses. Our results showed that CCCP did not alter the distribution and relative intensity of mitochondrial membrane potentials in Hipk $2^{+/+}$and Hipk2 ${ }^{-1-}$ MEFs at $2 \mathrm{~h}$ after treatment. Interestingly, beginning at $6 \mathrm{~h}$, and more prominently at $24 \mathrm{~h}$, CCCP treatment increased MitoTracker Red and MitoTracker Green staining intensity in $23-30 \%$ of $H i p k 2^{+/+}$ MEFs (Fig. 5B,C). In contrast, only $12-17 \%$ of Hipk2 ${ }^{-1-}$ MEFs showed similar changes at 6 and $24 \mathrm{~h}$. Consistent with these results, double-immunofluorescent confocal microscopy revealed that whereas CCCP treatment induced the dissociation of cytochrome C and Tom 20 in $>50 \%$ of Hipk ${ }^{+/+}$MEFs (Fig. 5D, arrowheads), only $\sim 15 \%$ of Hipk $2^{-1-}$ MEFs showed similar dissociation of cytochrome $\mathrm{C}$ from their mitochondria (Fig. $5 D, E)$. These results suggest that mitochondria in Hipk $2^{-1-}$ MEFs were more resistant to CCCP-induced changes in mitochondrial membrane potentials and integrity.

\section{Upregulation of Parkin target PGC-1 $\alpha$ in Hipk2 ${ }^{-1-}$ brain and MEF}

Previous studies have revealed several target genes downstream of Parkin, including PGC- $1 \alpha$, which is a transcriptional coactivator that regulates a number of genes that have important roles in mitochondrial functions (Lin et al., 2005; St-Pierre et al., 2006; Shin et al., 2011). Given the increase in Parkin protein levels in Hipk $2^{-1-}$ neurons and MEFs, we asked whether PGC- $1 \alpha$ is up- 

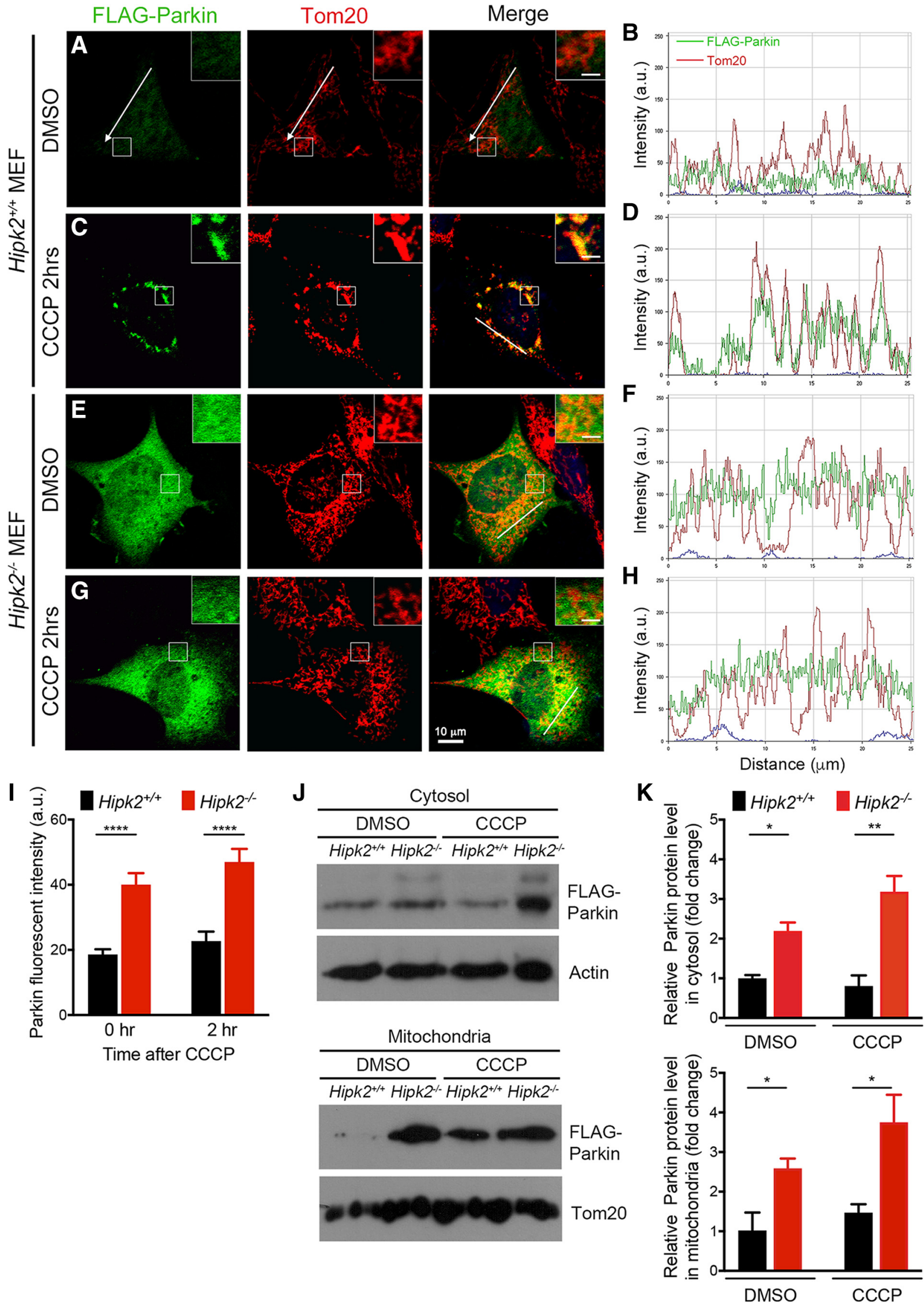

Figure 4. Hipk $2^{-1-}$ MEFs exhibit higher Parkin protein levels in the cytosol and mitochondria. $\boldsymbol{A}-\boldsymbol{H}$, Immunofluorescent confocal microscopic images highlight the relatively higher Parkin protein levels in control DMSO-treated and CCCP-treated Hipk2 ${ }^{-1-}$ MEFs. Both Hipk2 ${ }^{+/+}$and Hipk2 ${ }^{-1-}$ MEFs were transfected with constructs expressing FLAG-Parkin, treated with DMSO or СCCP $(5 \mu \mathrm{m})$ for $2 \mathrm{~h}$, fixed in 4\% PFA for immunostaining using anti-FLAG and anti-Tom20 antibodies, and processed for image analyses using the Nikon C2 (Figure legend continues.) 
regulated in these cells. In support of this idea, immunofluorescent confocal microscopy showed that most DA neurons in the substantia nigra and neurons in the motor cortex in Hipk2 $2^{-1-}$ mice had stronger signal intensity for PGC- $1 \alpha$ (Fig. $6 A, B)$. These results were further validated using Western blots that showed a higher abundance of PGC- $1 \alpha$ proteins in the substantia nigra and cortex of Hipk $2^{-1-}$ mice and in Hipk2 ${ }^{-1-}$ MEFs (Fig. 6C,D). Given the role of PGC- $1 \alpha$ as a transcriptional coactivator of genes that regulate mitochondrial biogenesis, these results suggested that the elevated PGC- $1 \alpha$ levels in Hipk $2^{-1-}$ neurons and MEFs most likely provide additional protection from mitochondrial toxicity.

\section{Discussion}

Results from this study show that HIPK2 can affect the proteasomemediated degradation of Parkin. The loss of HIPK2 in neurons and MEFs results in higher and saturated Parkin protein levels in the cytosol and mitochondria at basal growth conditions and after the uncoupling of mitochondrial membrane. These conditions protect neurons and MEFs from toxicity caused by mitochondrial toxins, perhaps through protecting mitochondria from injury, rapid recovery of damaged mitochondria, or both. While the exact mechanism for how HIPK2 regulates Parkin protein turnover remains unclear, several previous studies have implicated HIPK2 in post-translational modification of proteins via acetylation- and SUMO (small ubiquitin-like molecule)mediated degradation pathways. For instance, HIPK2 can phosphorylate E3 ubiquitin ligases SIAH1 and SIAH2, which are the mammalian homologs of the Drosophila gene seven in absentia that regulate the activity of tumor suppressor p53 (Grishina et al., 2012). Furthermore, elevated ROS can enhance HIPK2 acetylation and promote cell death (de la Vega et al., 2012). These results support the idea that HIPK2 most likely functions in a highly interconnected regulatory loop where HIPK2 and its interacting partners regulate the turnover of proteins critical for survival and cell death.

Of the three members in the HIPK family, HIPK1 is localized mostly in the cytoplasm and HIPK3 mostly in the nucleus, whereas HIPK2 can be detected in both compartments (Ritter and Schmitz, 2019). These results further support the expanding role of HIPK2 in regulating survival and cell death mechanisms via transcriptional machinery in both the nucleus and proteasome pathways in the cytoplasm. In light of these results, it is interesting to note that exposure to the chemical stressor sodium arsenite leads to a rapid and complete translocation of HIPK2

\footnotetext{
$\leftarrow$

(Figure legend continued.) Confocal Microscope. Insets in $\boldsymbol{A}, \boldsymbol{C}, \boldsymbol{E}$, and $\mathbf{G}$ represent highermagnification images of the highlighted areas. Scale bars: $A, C, E, G, 10 \mu \mathrm{m}$; insets, $2.5 \mu \mathrm{m}$. FLAG-Parkin and Tom20 immunofluorescent intensities were measured using Nikon NISElements software by drawing a line across the cytoplasm of Hipk2 ${ }^{+/+}$and Hipk2 ${ }^{-1-}$ MEFs (white lines in the "merge" in $A, C, E$, and $\boldsymbol{G}$ ). The signal intensity of Parkin and Tom 20 and the extent of their colocalization were presented in the corresponding panels in $\boldsymbol{B}, \boldsymbol{D}, \boldsymbol{F}$, and $\boldsymbol{H}$, where arrowheads indicate the colocalization of Parkin and Tom20. I, Quantification of overall Parkin immunofluorescent signal intensity in $\mathrm{Hipk2}^{+/+}$and Hipk2 ${ }^{-1-} \mathrm{MEFs}(\boldsymbol{A}, \boldsymbol{C}, \boldsymbol{E}$, and $\boldsymbol{G})$. Data represented the mean \pm SEM from 28 to 32 Parkin-expressing Hipk2 ${ }^{+/+}$and Hipk2 ${ }^{-1-}$ MEFs from four independent biological replicates. Statistical analyses used the Student's $t$ test: ${ }^{* * * *} p<0.001$. J, Western blot analysis of Parkin protein levels, detected by anti-FLAG antibody, in the cytosol and mitochondria of Hipk2 $2^{+/+}$and Hipk2 ${ }^{-1-}$ MEFs before and after CCCP treatment. Antibodies for actin and Tom 20 were used as loading controls for cytosolic and mitochondrial fractions, respectively. $\boldsymbol{K}$, Quantification of Parkin protein levels in the cytosol and mitochondria of Hipk2 ${ }^{+/+}$and Hipk2 ${ }^{-1-}$ MEFs before and after CCCP treatment. Data represented the mean \pm SEM from three independent biological replicates. Statistical analyses used the Student's $t$ test: ${ }^{*} p<0.05$ and ${ }^{* *} p<0.01$.
}

to the nucleus. It is conceivable that such dynamic regulation of HIPK2 in different subcellular compartments may be coupled with stabilization of potential HIPK2-interacting partners, such as Parkin, that can have an impact on survival and cell death.

Mutations in Parkin are associated with familial Parkinson's disease. Indeed, most functional characterizations of Parkin have provided strong evidence supporting the role of Parkin in repairing damaged mitochondria via mitophagy, a form of selective autophagy (Narendra and Youle, 2011). Interestingly, Parkindeficient mice do not show any detectable degeneration in DA neurons (Goldberg et al., 2003; Von Coelln et al., 2004). By contrast, overexpression of wild-type Parkin in neurons using lentivirus or a transgenic approach protects DA neurons from toxicity caused by mutant $\alpha$-synuclein or MPTP, respectively (Lo Bianco et al., 2004; Bian et al., 2012). Consistent with these results, the robust upregulation of Parkin protein levels in Hipk2 ${ }^{-1-}$ mouse brain tissues and MEFs provide supporting evidence that Hipk2 ${ }^{-1-}$ neurons and MEFs are indeed much more resistant to mitochondrial toxins, such as MPTP, rotenone, and paraquat.

While most attention on Parkin has focused on its role in mitophagy, there is evidence that Parkin is involved in several nonmitophagy mechanisms. For instance, Parkin ubiquitinates the misfolded PAEL receptor in the ER and promotes the degradation of PAEL receptors (Imai et al., 2001). This presumably alleviates ER stress induced by the accumulation of misfolded PAEL receptors. In addition, Parkin can also interact with the transcriptional repressor PARIS, which suppresses the expression of transcriptional coactivator PGC- $1 \alpha$ and its downstream target genes that can promote mitochondrial biogenesis (Shin et al., 2011). Finally, using a small interfering RNA screen combined with high-content microscopy, Hasson et al. (2013) identified several candidates that are involved in diverse cellular processes that could influence the Parkin signaling pathway, including the mitochondrial protein TOMM7 and heat shock protein HSPA1L1.

The implication of HIPK2 in regulating proteasome-mediated Parkin protein degradation raises the question as to whether HIPK2 could be a part of signaling pathways that function upstream of Parkin. In support of this idea, several other kinases have been implicated in Parkin-mediated resistance to neurodegeneration. For example, exposure to neurotoxin MPTP activates nonreceptor tyrosine kinase c-Abl, which phosphorylates Parkin on tyrosine residue at position 143. This inactivates the ubiquitin E3 ligase activity in Parkin and mitigates its neuroprotective function (Ko et al., 2010). Perhaps most relevant to our current study is the documented role of JNK in Parkin-mediated signaling pathway. Overexpression of Parkin in neuroblastoma cells significantly attenuates the activation of JNK and caspase 3, and protects cells from toxicity caused by dopamine, 6-OHDA, or microtubule-depolarizing toxins (Jiang et al., 2004; Ren et al., 2009). In another study, DA neurons in Parkin loss-of-function Drosophila mutants exhibit prominent activation of JNK, which directly contributes to neurodegenerative features and behavioral phenotypes in these mutants (Cha et al., 2005). Given the role of HIPK2 and JNK in neurodegeneration induced by ER stress and in mouse models of Alzheimer's disease and amyotrophic lateral sclerosis (Lee et al., 2016; Le Pichon et al., 2017), it is conceivable that HIPK2 and Parkin may have mutual regulation in response to neurodegenerative conditions. Consistent with this idea, previous studies have shown that Parkin and PINK1 can respond to the unfolded protein response in the mitochondria to mitigate proteostasis-induced mitochondrial 
A

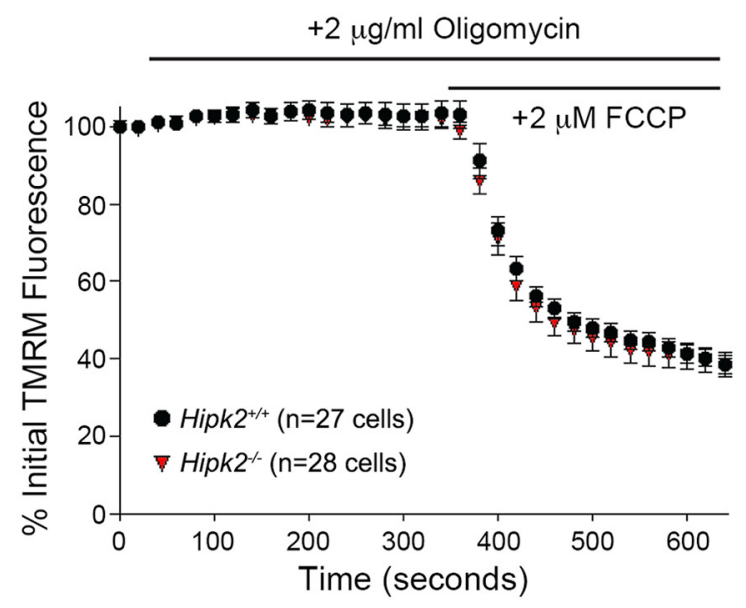

C

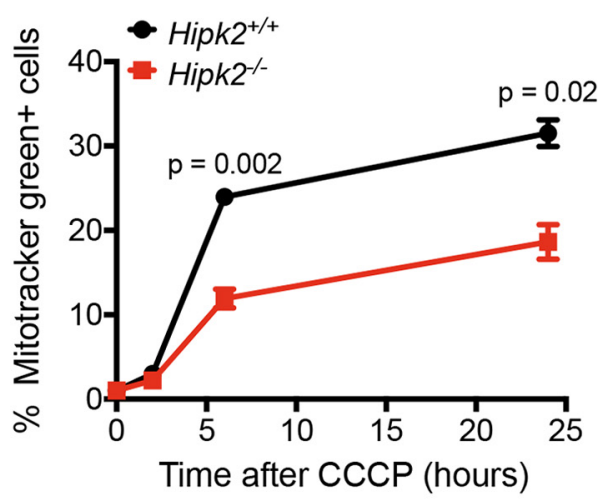

E

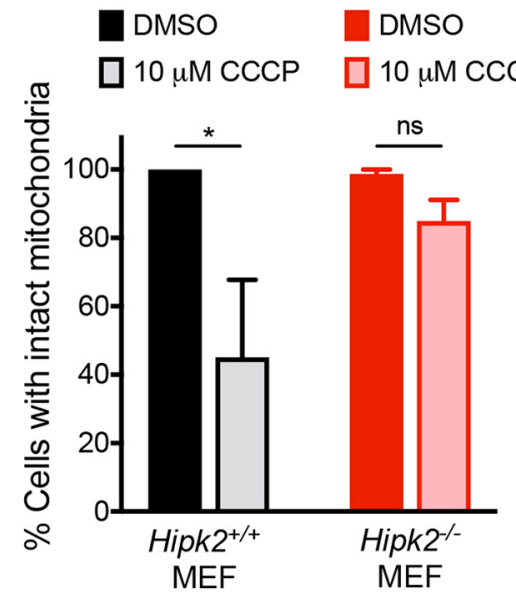

B

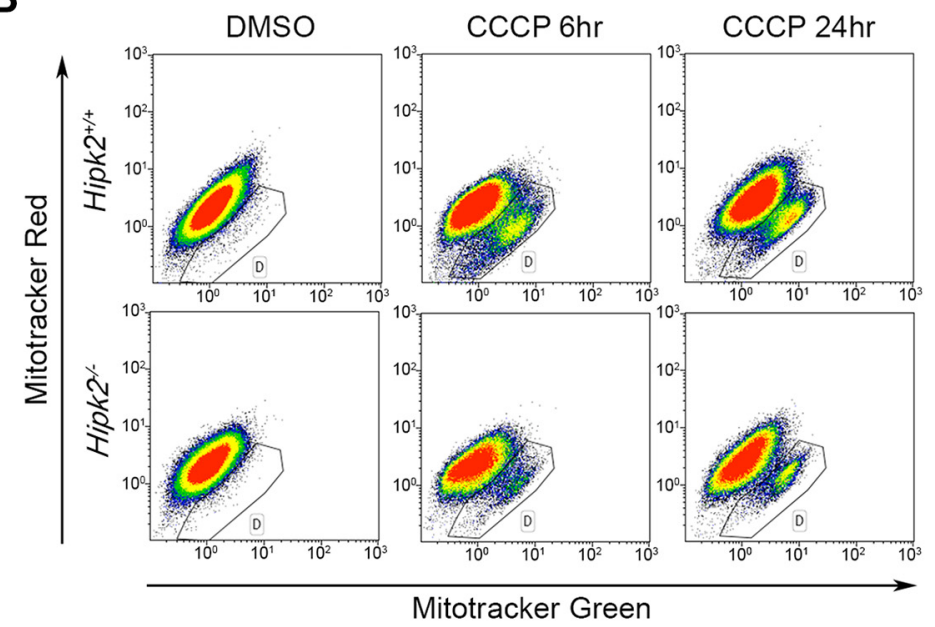

D
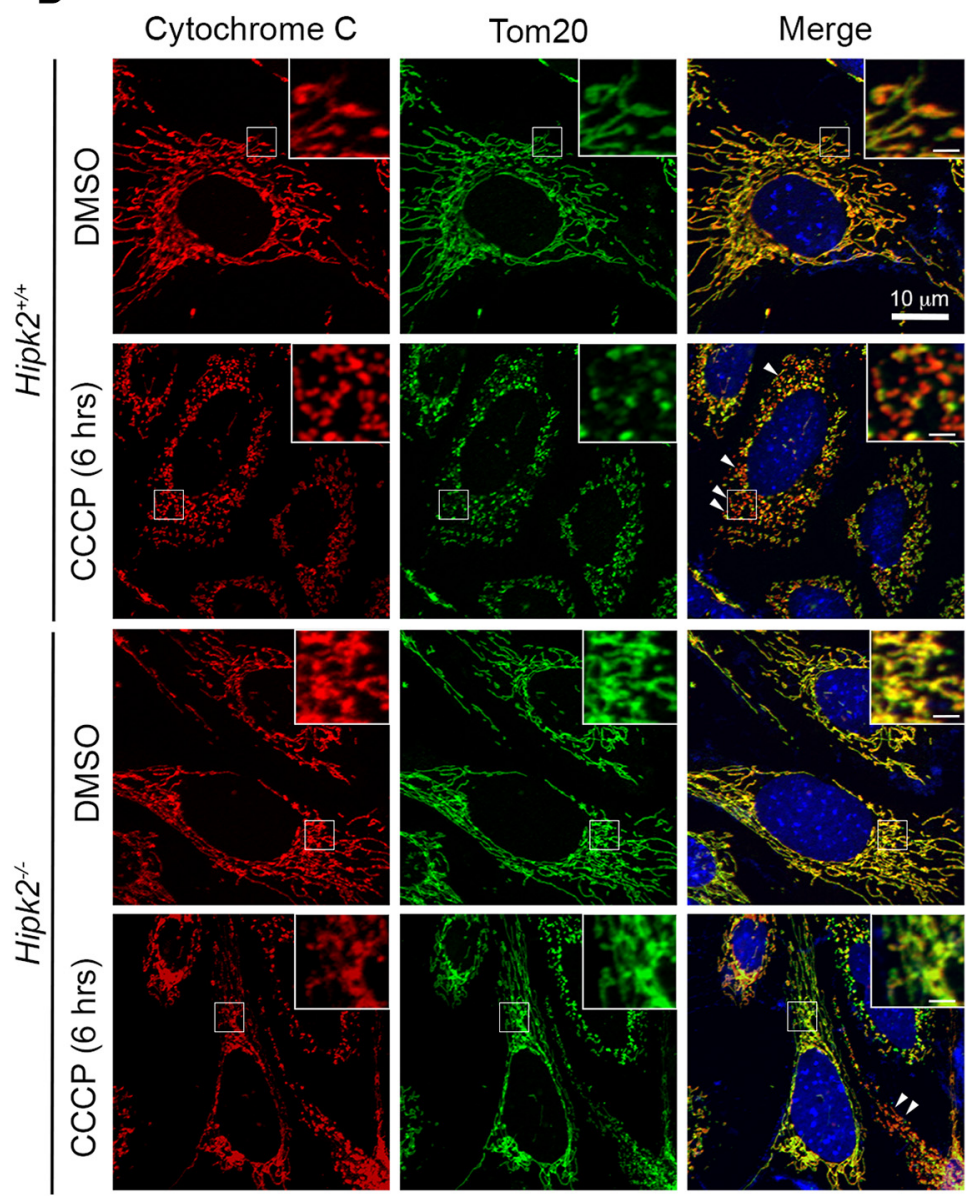

Figure 5. CCCP-treated Hipk2 ${ }^{-1-}$ MEFs show more intact mitochondria, but no change in mitochondrial membrane potentials. A, Mitochondrial membrane potentials in Hipk2 ${ }^{+/+}$and Hipk2 ${ }^{-1-}$ MEFs were measured by TMRM dye. Hipk2 $2^{+/+}$and Hipk2 ${ }^{-1-}$ MEFs were treated sequentially with $2 \mu \mathrm{g} / \mathrm{ml}$ oligomycin and $2 \mu \mathrm{m} \mathrm{FCCP} \mathrm{to} \mathrm{inhibit} \mathrm{ATP} \mathrm{synthase} \mathrm{in} \mathrm{complex} \mathrm{V} \mathrm{and} \mathrm{proton}$ gradient, respectively. B, C, FACS data from Hipk $2^{+/+}$and Hipk2 $2^{-1-}$ MEFs, which were preloaded with MitoTracker-Red and MitoTracker-Green to measure mitochondrial membrane potentials and mitochondrial mass, respectively. Hipk2 ${ }^{+/+}$and Hipk2 ${ }^{-/-}$MEFs were then treated with DMSO (control) or $10 \mu \mathrm{m} \mathrm{CCCP} \mathrm{for} \mathrm{2,} \mathrm{6,} \mathrm{or} 24 \mathrm{~h}$ and collected for FACS analysis. Cells with higher MitoTracker-Green signals were gated for further quantification, and the results are presented in $\boldsymbol{B}$. Data represented the mean \pm SEM from three independent biological replicates. Statistical analyses used the Student's test. D, E, Hipk2 $2^{+/+}$and Hipk2 $2^{-1-}$ MEFs were used in immunofluorescent microscopy using anti-cytochrome C and anti-Tom20 antibodies to determine the integrity of mitochondria under control condition or after membrane uncoupling by CCCP treatment. Cells with mitochondria that showed positive cytochrome $C$ and Tom 20 signals were scored as having intact mitochondrial membrane, whereas those with positive cytochrome C but no Tom20 signal were scored as having no intact mitochondria (highlighted by arrowheads). Insets in $\boldsymbol{D}$ represent higher-magnification images of the highlighted areas. Scale bars: inset, $2.5 \mu \mathrm{m}$. The percentage of Hipk2 ${ }^{+/+}$and Hipk $2^{-1-}$ MEFs with intact mitochondrial membrane was shown in $\boldsymbol{E}$. Data represented the mean \pm SEM from $30 \mathrm{Hipk2}^{+/+}$and Hipk2 ${ }^{-/-}$MEFs from three independent biological replicates. Statistical analyses used the Student's $t$ test: ns, not significant; ${ }^{*} p<0.05$. 
A
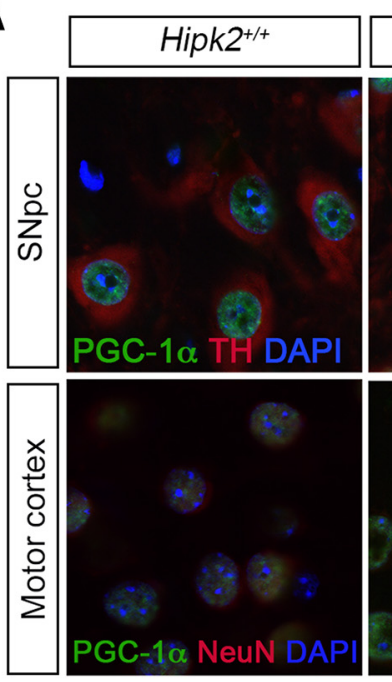
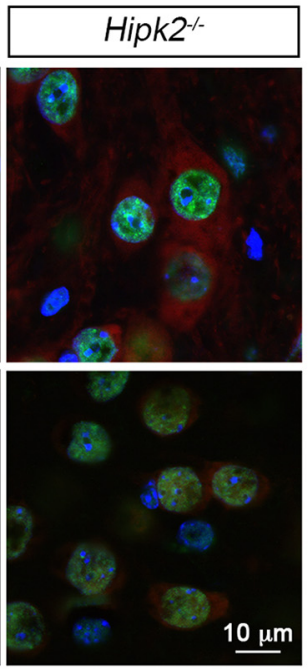

B

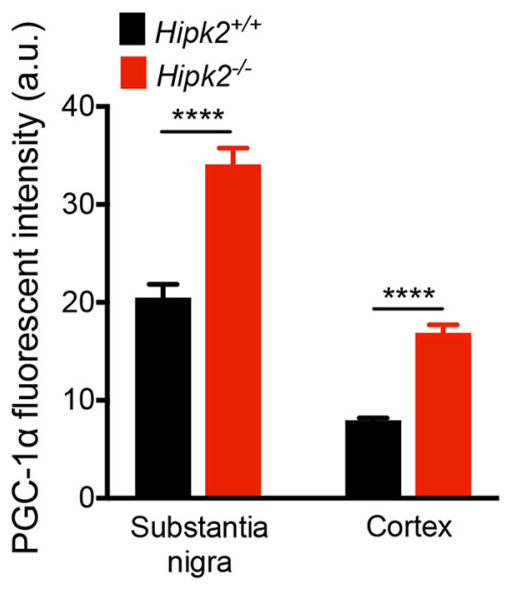

C SNpc

Cortex

MEF

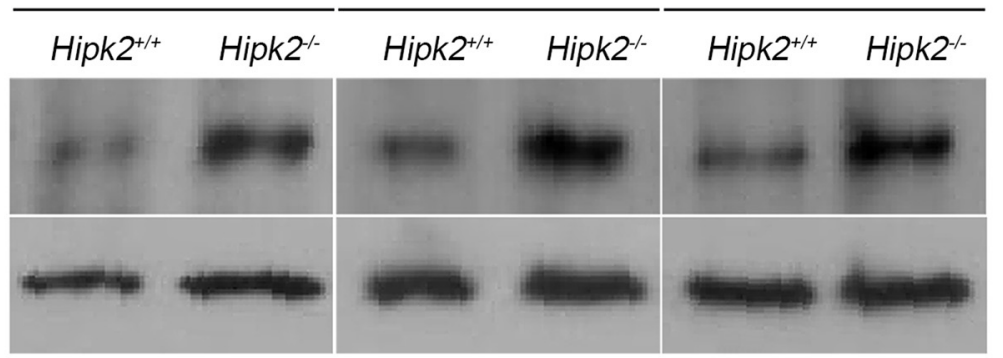

PGC-1 $\alpha$

Actin

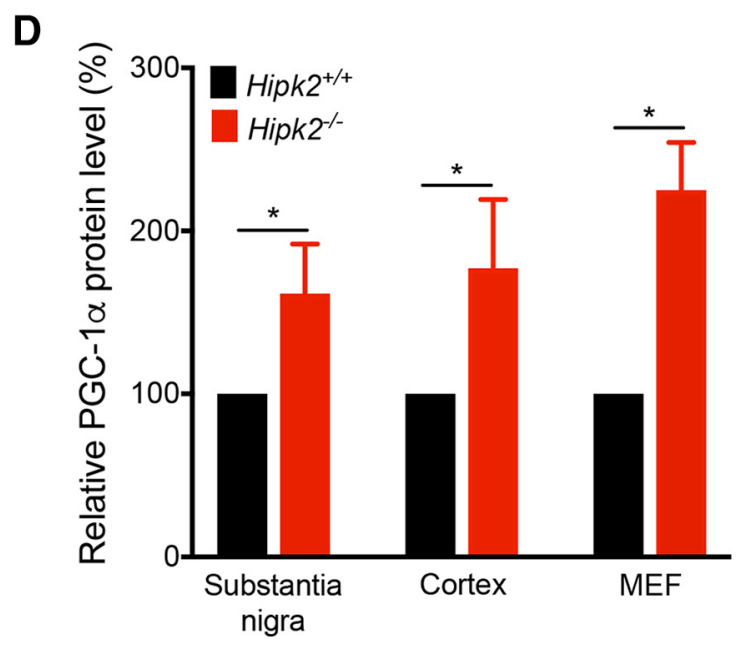

Figure 6. Upregulation of PGC $-1 \alpha$ in Hipk2 ${ }^{-1-}$ neurons and MEFs. A, Immunofluorescent confocal microscopic images of PGC $-1 \alpha$ expression in substantia nigra DA neurons (labeled by anti-TH antibody) and cortical neurons (labeled by anti-NeuN antibody) in Hipk2 $2^{+/+}$and Hipk2 $2^{-1-}$ mice. $\boldsymbol{B}, \mathrm{PGC}-1 \alpha$ protein immunofluorescence intensity in DA neurons and cortical neurons was quantified using NIH ImageJ. Data represented the mean \pm SEM from $40 \mathrm{Hipk2^{+/+ }}$ and Hipk2 ${ }^{-1-}$ DA neurons and cortical neurons from four independent biological replicates. Statistical analyses used the Student's $t$ test: ${ }^{* * *} p<0.01$. C, D, Western blot analysis of PGC $-1 \alpha$ protein levels in lysates from Hipk ${ }^{+/+}$and Hipk2 ${ }^{-1-}$ substantia nigra, cerebral cortex, and MEFs. Antibody for actin was used as the loading control. Data represented the mean \pm SEM from four independent biological replicates. Statistical analyses used the Student's $t$ test: ${ }^{*} p<0.05$.

damage (Burman et al., 2017). Finally, the role of HIPK2 in Parkinmediated protection of mitochondrial integrity and neuronal survival may depend on other non-proteasome-dependent mechanisms. Future experiments should aim to uncover whether and how HIPK2 may directly or indirectly interact with other partners that could regulate its proteasome-mediated degradation of Parkin.
In summary, the results from this study reveal the previously unappreciated role of HIPK2 in regulating Parkin protein level via the proteasome-mediated pathway. The elevated Parkin protein levels in Hipk2 $2^{-1-}$ neurons and MEFs contribute to the resistance of these cells to the toxicity caused by mitochondrial toxins. 


\section{References}

Bian M, Liu J, Hong X, Yu M, Huang Y, Sheng Z, Fei J, Huang F (2012) Overexpression of parkin ameliorates dopaminergic neurodegeneration induced by 1-methyl-4-phenyl-1,2,3,6-tetrahydropyridine in mice. PLoS One 7:e39953.

Blaquiere JA, Verheyen EM (2017) Homeodomain-interacting protein kinases: diverse and complex roles in development and disease. Curr Top Dev Biol 123:73-103.

Burman JL, Pickles S, Wang C, Sekine S, Vargas JNS, Zhang Z, Youle AM, Nezich CL, Wu X, Hammer JA, Youle RJ (2017) Mitochondrial fission facilitates the selective mitophagy of protein aggregates. J Cell Biol 216:3231-3247.

Castello PR, Drechsel DA, Patel M (2007) Mitochondria are a major source of paraquat-induced reactive oxygen species production in the brain. J Biol Chem 282:14186-14193.

Cha GH, Kim S, Park J, Lee E, Kim M, Lee SB, Kim JM, Chung J, Cho KS (2005) Parkin negatively regulates JNK pathway in the dopaminergic neurons of Drosophila. Proc Natl Acad Sci U S A 102:10345-10350.

Chipuk JE, Green DR (2008) How do BCL-2 proteins induce mitochondrial outer membrane permeabilization? Trends Cell Biol 18:157-164.

de la Vega L, Grishina I, Moreno R, Krüger M, Braun T, Schmitz ML (2012) A redox-regulated SUMO/acetylation switch of HIPK2 controls the survival threshold to oxidative stress. Mol Cell 46:472-483.

Eckert A, Schulz KL, Rhein V, Götz J (2010) Convergence of amyloid-beta and tau pathologies on mitochondria in vivo. Mol Neurobiol 41:107-114.

Fan Y, Wang N, Chuang P, He JC (2014) Role of HIPK2 in kidney fibrosis. Kidney Int Suppl (2011) 4:97-101.

Goldberg MS, Fleming SM, Palacino JJ, Cepeda C, Lam HA, Bhatnagar A, Meloni EG, Wu N, Ackerson LC, Klapstein GJ, Gajendiran M, Roth BL, Chesselet MF, Maidment NT, Levine MS, Shen J (2003) Parkindeficient mice exhibit nigrostriatal deficits but not loss of dopaminergic neurons. J Biol Chem 278:43628-43635.

Grishina I, Debus K, García-Limones C, Schneider C, Shresta A, García C, Calzado MA, Schmitz ML (2012) SIAH-mediated ubiquitination and degradation of acetyl-transferases regulate the $\mathrm{p} 53$ response and protein acetylation. Biochim Biophys Acta 1823:2287-2296.

Hasson SA, Kane LA, Yamano K, Huang CH, Sliter DA, Buehler E, Wang C, Heman-Ackah SM, Hessa T, Guha R, Martin SE, Youle RJ (2013) Highcontent genome-wide RNAi screens identify regulators of parkin upstream of mitophagy. Nature 504:291-295.

Hofmann TG, Glas C, Bitomsky N (2013) HIPK2: a tumour suppressor that controls DNA damage-induced cell fate and cytokinesis. Bioessays 35:55-64.

Imai Y, Soda M, Inoue H, Hattori N, Mizuno Y, Takahashi R (2001) An unfolded putative transmembrane polypeptide, which can lead to endoplasmic reticulum stress, is a substrate of parkin. Cell 105:891-902.

Israelson A, Arbel N, Da Cruz S, Ilieva H, Yamanaka K, Shoshan-Barmatz V, Cleveland DW (2010) Misfolded mutant SOD1 directly inhibits VDAC1 conductance in a mouse model of inherited ALS. Neuron 67:575-587.

Jiang H, Ren Y, Zhao J, Feng J (2004) Parkin protects human dopaminergic neuroblastoma cells against dopamine-induced apoptosis. Hum Mol Genet 13:1745-1754.

Klein C, Westenberger A (2012) Genetics of Parkinson's disease. Cold Spring Harb Perspect Med 2:a008888.

Ko HS, Lee Y, Shin JH, Karuppagounder SS, Gadad BS, Koleske AJ, Pletnikova O, Troncoso JC, Dawson VL, Dawson TM (2010) Phosphorylation by the $\mathrm{c}$-abl protein tyrosine kinase inhibits parkin's ubiquitination and protective function. Proc Natl Acad Sci U S A 107:16691-16696.

Le Pichon CE, Meilandt WJ, Dominguez S, Solanoy H, Lin H, Ngu H, Gogineni A, Sengupta Ghosh A, Jiang Z, Lee SH, Maloney J, Gandham VD, Pozniak CD, Wang B, Lee S, Siu M, Patel S, Modrusan Z, Liu X, Rudhard $\mathrm{Y}$, et al. (2017) Loss of dual leucine zipper kinase signaling is protective in animal models of neurodegenerative disease. Sci Transl Med 9:eaag0394.

Lee S, Shang Y, Redmond SA, Urisman A, Tang AA, Li KH, Burlingame AL, Pak RA, Jovičić A, Gitler AD, Wang J, Gray NS, Seeley WW, Siddique T, Bigio EH, Lee VM, Trojanowski JQ, Chan JR, Huang EJ (2016) Activation of HIPK2 promotes ER stress-mediated neurodegeneration in amyotrophic lateral sclerosis. Neuron 91:41-55.

Li N, Ragheb K, Lawler G, Sturgis J, Rajwa B, Melendez JA, Robinson JP (2003) Mitochondrial complex I inhibitor rotenone induces apoptosis through enhancing mitochondrial reactive oxygen species production. J Biol Chem 278:8516-8525.

Lin J, Handschin C, Spiegelman BM (2005) Metabolic control through the PGC-1 family of transcription coactivators. Cell Metab 1:361-370.

Lindeberg J, Usoskin D, Bengtsson H, Gustafsson A, Kylberg A, Söderström S, Ebendal T (2004) Transgenic expression of cre recombinase from the tyrosine hydroxylase locus. Genesis 40:67-73.

Lo Bianco C, Schneider BL, Bauer M, Sajadi A, Brice A, Iwatsubo T, Aebischer $P$ (2004) Lentiviral vector delivery of parkin prevents dopaminergic degeneration in an alpha-synuclein rat model of Parkinson's disease. Proc Natl Acad Sci U S A 101:17510-17515.

Luo SX, Timbang L, Kim JI, Shang Y, Sandoval K, Tang AA, Whistler JL, Ding JB, Huang EJ (2016) TGF-beta signaling in dopaminergic neurons regulates dendritic growth, excitatory-inhibitory synaptic balance, and reversal learning. Cell Rep 17:3233-3245.

Matsuda N, Sato S, Shiba K, Okatsu K, Saisho K, Gautier CA, Sou YS, Saiki S, Kawajiri S, Sato F, Kimura M, Komatsu M, Hattori N, Tanaka K (2010) PINK1 stabilized by mitochondrial depolarization recruits parkin to damaged mitochondria and activates latent parkin for mitophagy. J Cell Biol 189:211-221.

Mendelsohn BA, Bennett NK, Darch MA, Yu K, Nguyen MK, Pucciarelli D, Nelson M, Horlbeck MA, Gilbert LA, Hyun W, Kampmann M, Nakamura JL, Nakamura K (2018) A high-throughput screen of real-time ATP levels in individual cells reveals mechanisms of energy failure. PLoS Biol 16:e2004624.

Nakamura K, Nemani VM, Azarbal F, Skibinski G, Levy JM, Egami K, Munishkina L, Zhang J, Gardner B, Wakabayashi J, Sesaki H, Cheng Y, Finkbeiner S, Nussbaum RL, Masliah E, Edwards RH (2011) Direct membrane association drives mitochondrial fission by the Parkinson disease-associated protein alpha-synuclein. J Biol Chem 286:20710-20726.

Narendra DP, Youle RJ (2011) Targeting mitochondrial dysfunction: role for PINK1 and parkin in mitochondrial quality control. Antioxid Redox Signal 14:1929-1938.

Narendra D, Tanaka A, Suen DF, Youle RJ (2008) Parkin is recruited selectively to impaired mitochondria and promotes their autophagy. J Cell Biol 183:795-803.

Ren Y, Jiang H, Yang F, Nakaso K, Feng J (2009) Parkin protects dopaminergic neurons against microtubule-depolymerizing toxins by attenuating microtubule-associated protein kinase activation. J Biol Chem 284: 4009-4017.

Riedl SJ, Salvesen GS (2007) The apoptosome: signalling platform of cell death. Nat Rev Mol Cell Biol 8:405-413.

Ritter O, Schmitz ML (2019) Differential intracellular localization and dynamic nucleocytoplasmic shuttling of homeodomain-interacting protein kinase family members. Biochim Biophys Acta Mol Cell Res 1866:1676-1686.

Shang Y, Zhang J, Huang EJ (2018) HIPK2-mediated transcriptional control of NMDA receptor subunit expression regulates neuronal survival and cell death. J Neurosci 38:4006-4019.

Sheng ZH, Cai Q (2012) Mitochondrial transport in neurons: impact on synaptic homeostasis and neurodegeneration. Nat Rev Neurosci 13: 77-93.

Shin JH, Ko HS, Kang H, Lee Y, Lee YI, Pletinkova O, Troconso JC, Dawson VL, Dawson TM (2011) PARIS (ZNF746) repression of PGC-lalpha contributes to neurodegeneration in Parkinson's disease. Cell 144:689-702.

St-Pierre J, Drori S, Uldry M, Silvaggi JM, Rhee J, Jager S, Handschin C, Zheng K, Lin J, Yang W, Simon DK, Bachoo R, Spiegelman BM (2006) Suppression of reactive oxygen species and neurodegeneration by the PGC-1 transcriptional coactivators. Cell 127:397-408.

Tang M, Miyamoto Y, Huang EJ (2009) Multiple roles of beta-catenin in controlling the neurogenic niche for midbrain dopamine neurons. Development 136:2027-2038.

Tang M, Villaescusa JC, Luo SX, Guitarte C, Lei S, Miyamoto Y, Taketo MM, Arenas E, Huang EJ (2010) Interactions of Wnt $/ \beta$-catenin signaling and sonic hedgehog regulate the neurogenesis of ventral midbrain dopamine neurons. J Neurosci 30:9280-9291.

Vives-Bauza C, Zhou C, Huang Y, Cui M, de Vries RL, Kim J, May J, Tocilescu MA, Liu W, Ko HS, Magrané J, Moore DJ, Dawson VL, Grailhe R, Dawson TM, Li C, Tieu K, Przedborski S (2010) PINK1-dependent recruitment of parkin to mitochondria in mitophagy. Proc Natl Acad Sci U S A 107: $378-383$. 
Von Coelln R, Thomas B, Savitt JM, Lim KL, Sasaki M, Hess EJ, Dawson VL, Dawson TM (2004) Loss of locus coeruleus neurons and reduced startle in parkin null mice. Proc Natl Acad Sci U S A 101:1074410749.

Wang W, Wang L, Lu J, Siedlak SL, Fujioka H, Liang J, Jiang S, Ma X, Jiang Z, da Rocha EL, Sheng M, Choi H, Lerou PH, Li H, Wang X (2016) The inhibition of TDP-43 mitochondrial localization blocks its neuronal toxicity. Nat Med 22:869-878.

Wei G, Ku S, Ma GK, Saito S, Tang AA, Zhang J, Mao JH, Appella E, Balmain A, Huang EJ (2007) HIPK2 represses beta-catenin-mediated transcription, epidermal stem cell expansion, and skin tumorigenesis. Proc Natl Acad Sci U S A 104:13040-13045.

Wiggins AK, Wei G, Doxakis E, Wong C, Tang AA, Zang K, Luo EJ, Neve RL, Reichardt LF, Huang EJ (2004) Interaction of Brn3a and HIPK2 medi- ates transcriptional repression of sensory neuron survival. J Cell Biol 167:257-267.

Youle RJ, Strasser A (2008) The BCL-2 protein family: opposing activities that mediate cell death. Nat Rev Mol Cell Biol 9:47-59.

Youle RJ, van der Bliek AM (2012) Mitochondrial fission, fusion, and stress. Science 337:1062-1065.

Zhang J, Pho V, Bonasera SJ, Holtzman J, Tang AT, Hellmuth J, Tang S, Janak PH, Tecott LH, Huang EJ (2007) Essential function of HIPK2 in TGFbeta-dependent survival of midbrain dopamine neurons. Nat Neurosci 10:77-86.

Zheng L, Bernard-Marissal N, Moullan N, D’Amico D, Auwerx J, Moore DJ, Knott G, Aebischer P, Schneider BL (2017) Parkin functionally interacts with PGC-1alpha to preserve mitochondria and protect dopaminergic neurons. Hum Mol Genet 26:582-598. 